\title{
Study of Instabilities on Laminar-Turbulent Transition in a 4 Lug-Bolt in a Confined Diffusion Flame
}

\author{
Mauricio De la Cruz ${ }^{1}$, Eliseo Martínez-Espinosa ${ }^{2}$, Georgiy Polupan ${ }^{1}$ \\ ${ }^{1}$ Instituto Politécnico Nacional, Laboratorio de Ingeniería Térmica e Hidráulica Aplicada, Unidad Profesional Zacatenco \\ Edif. 5, 3er piso, C.P. 07738 México D.F., MÉXICO. \\ mauriciodlca@hotmail.com; gpolupan85@yahoo.com.mx \\ ${ }^{2}$ Instituto de Ingeniería, Universidad Nacional Autónoma de México Ciudad Universitaria \\ 04510 México D.F, MÉXICO \\ emartineze@iingen.unam.mx
}

\begin{abstract}
Velocity fluctuation in a diffusion methane-oxygen flame for three different injection velocities is analyzed numerically in this paper. The work states a 4 Lug-Bolt arrange where oxygen is injected by a central nozzle and methane by four peripheral nozzles. The aim of the numerical simulation is to study the mechanism that affect the mixture process in a diffusion flame by means of KelvinHelmholtz instabilities. The simulation is realized with the Reynolds-Averaged Navier-Stokes technique and the realizable k- $\varepsilon$ turbulence model is considered in order to model the Reynolds stress tensor. The results show a recirculation zone which is the essential mixture mechanism and has relevant participation on the instabilities development in confined diffusion flames. Furthermore, predictions exhibit a wave frequency increment and wave length instability decrement as velocity injection increase. Therefore, in diffusion flames the Kelvin-Helmholtz instability affects the mixing and reaction zone.
\end{abstract}

Keywords: Diffusion flame, Fluctuating velocity, Numerical simulation, Kelvin-Helmholtz instability

\section{Introduction}

The analyses of fluctuating velocity in combustion processes show a dependency upon the mixture composition and the coherent vortices are located in the outer part of flame with the trajectory of their centers moving far from the flame front. Their location and large sizes (comparable with the flow width) suggest their important role in mixing and, especially, in the entrainment processes which are essential in combustion systems. For example, Katta and Roquemore [1] observed a vortices decay into turbulence in axial location, approximately 20 diameters from the nozzle exit, which is a far larger distance than in isothermal flows. The reason of such long-distance of coherence is probably the great increase of fluid viscosity resulting in flow laminarization due to high temperatures [1]. In diffusion flames, a second type of organized vortex structures dominates on the outer structure of the jet and the mixing intense. The most probable mechanism of generation of the outer structures is a Kelvin-Helmholtz $(\mathrm{KH})$ instability [2, 3]. Free jets with chemical reaction are very difficult to investigate therefore a few experimental investigations are in open literature so far. The only evidence in the literature confirming the origin of outer structures are the results of a numerical-experimental study performed by Davis et al [4] and. R. D. Hancock et al [5]. The authors have applied a simple combustion model and variations of the velocity and temperature fields in a propane jet. Therefore, the current numerical study is aimed to determinate detailed velocity fields in confined diffusion methane flame in a transition to turbulent flow. In order to organize the perturbations derived of the mixing zones and for better understanding of the perturbation origin. A shear velocity analysis is performed due its direct connection for the instability formation near the outer coherent structures.

\section{Combustion Kinetics}

The energy production by methane or natural gas combustion is well established [6] by the next overall reaction:

$$
\mathrm{CH}_{4}+2 \mathrm{O}_{2}=\mathrm{CO}_{2}+2 \mathrm{H}_{2} \mathrm{O}, \Delta \mathrm{H}_{298}=-802.7 \mathrm{~kJ} / \mathrm{mol}
$$


This overall equation is, however, a gross simplification with the actual reaction mechanism involving many free radical chain reactions [5]. Nevertheless the mean purpose of this work is not to analyze the secondary chemical reactions for this reason one step reaction was use. The eq. 1 is modelled as a stoichiometric reaction.

\section{Numerical Details}

The numerical simulation poses an oxygen-methane mixing process at atmospheric conditions in a combustion chamber. The analysis uses an oxygen injection through a central $16.9 \mathrm{~mm}$ nozzle diameter and methane through four peripheral $5.86 \mathrm{~mm}$ nozzles diameter, as shown in figure 2. The current distance between injector's axes is $33.87 \mathrm{~mm}$ (radial distance).This is a geometrical 4 x 67.74 Lug-Bolt array. The combustion chamber has a 101.6mm diameter and $1500 \mathrm{~mm}$ of total length. The aim of the numerical simulation is to calculate and analyze the mechanism affecting the mixture process of two incompressible flows under the Kelvin-Helmholtz instabilities and shear velocity fluctuations criteria. The work states three different injection velocities, for case A $2.5 \mathrm{~m} / \mathrm{s}, 5 \mathrm{~m} / \mathrm{s}$ for B and $10 \mathrm{~m} / \mathrm{s}$ for the case C. The simulations use the standard properties for methane and oxygen mixture species. The calculus domain has a non-slip boundary condition and adiabatic treatment for the calculus domain wall was use. A zero pressure gradient is considered for the outlet flow. To calculate the effect of turbulent chemical reaction rate The Eddy Dissipation Model (EDM) was used. A 3rd order Quadratic Upstream Interpolation for Convective Kinematics (Quick) for the convective and viscous terms was considered. Reynolds-Averaged Navier-Stokes (RANS) technique and realizable k- $\varepsilon$ turbulence model was used to model the Reynolds stress tensor. This turbulence model has been validated experimentally for many reactive flows with satisfactory results [7-10]. In combustion modeling, a density-weighted average or Favre average method was used. A mesh with, approximately, 1346298 cells was used. The numerical domain aspects are shown in figure 2 . This work allows to be applied to further studies thermo-acoustics combustion phenomena.
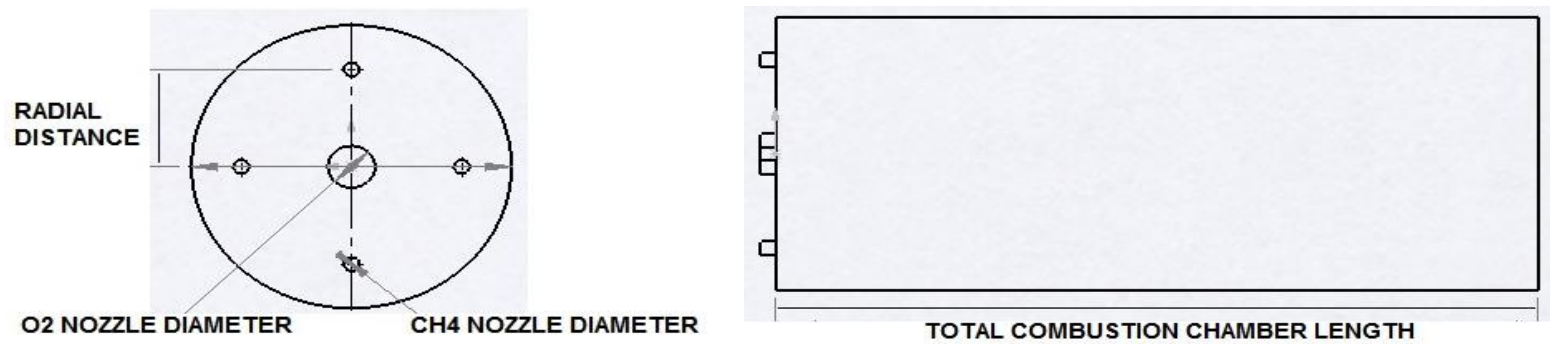

Fig. 2: Geometry and calculus domain.

\subsection{Constitutive Equations}

The equations to be solved are conservation of mass, momentum, energy and chemical species. The Reynoldsaveraged Navier-Stokes formulation is based on ensemble averaging and closure problem. The density-weighted averaging or the so-called Favre-averaging [11] is considered where the symbol ' $\sim$ ' denotes a density weighted ensemble average. The Favre-averaged continuity, momentum, energy and species equations are expressed as follows:

\section{Mass conservation:}

$$
\frac{\partial \bar{\rho}}{\partial t}+\nabla \cdot(\bar{\rho} \widetilde{\mathbf{v}})=0
$$

Momentum:

$$
\bar{\rho} \frac{\partial \tilde{\mathbf{v}}}{\partial t}+\bar{\rho} \tilde{\mathbf{v}} \cdot \nabla \tilde{\mathbf{v}}=-(\nabla \overline{\mathbf{p}})+\nabla \cdot \bar{\tau}+\bar{\rho} \sum_{i=1}^{N} \widetilde{Y_{l} \mathbf{f}_{l}}-\nabla\left(\bar{\rho} \widetilde{\mathbf{v}}^{\top} \mathbf{v}^{\prime}\right)
$$

Conservation of Energy:

Species:

$$
\bar{\rho} \frac{\partial \tilde{\boldsymbol{e}}}{\partial t}+\bar{\rho} \tilde{\mathbf{v}} \cdot \nabla \tilde{e}=-\nabla \cdot \widetilde{\mathbf{q}}-\overline{p \nabla \cdot \mathbf{v}}+\overline{\tau: \nabla \mathbf{v}}+\bar{\rho} \sum_{i=1}^{N} Y_{l} \overline{\mathbf{f}_{l} \cdot \mathbf{V}_{l}}-\nabla \cdot\left(\bar{\rho} \widetilde{v^{\top} e^{\prime}}\right)
$$

$$
\bar{\rho} \frac{\partial \tilde{Y}_{i}}{\partial t}+\bar{\rho} \tilde{\mathbf{v}} \cdot \nabla \widetilde{Y}_{l}=\nabla \cdot\left(-\bar{\rho} \widetilde{Y_{l} \widetilde{\mathbf{V}}_{l}}\right)+\widetilde{\widetilde{\omega}_{l}}-\nabla \cdot\left(\bar{\rho} \widetilde{\mathbf{v}}^{\top} Y_{l}^{\prime}\right), \quad i=1, \ldots, N
$$


where $\rho \widetilde{\mathbf{v}^{\top} \mathbf{v}^{\prime}}, \bar{\rho} \widetilde{\mathbf{v}^{\prime} Y^{\prime}}{ }_{l}$ and $\bar{\rho} \widetilde{\mathbf{v}^{\prime} e^{\prime}}$ are the apparent Reynolds stresses tensor, mass-weight density fluctuations, and turbulent heat transfer vector, respectively.

\section{Results}

Figure 3 shows the jets streamlines based on the axial and radial velocities, which exhibit a recirculation zone as is described by De la Cruz, et al. [12] with a 1L/Dc length for three cases. Fifty raster stream lines were used in order to have the same vortex visualization. For the case A, the injection velocity causes a low swirl traces due a drag and impulse movement driven by the central jet pressing the methane-oxygen mixture just before oxygen and methane jets. The drag impulses the methane to the oxygen mean flow, thereupon the methane velocity decreases and pressed to the chamber wall. The oxygen potential core is 2.3 times the methane potential core, this penetration length causes a rapid expansion over the methane jet hampering its development. This type of movement causes a toroidal swirl improving the species mixing process because injection velocity $(2.5 \mathrm{~m} / \mathrm{s})$ is near to the transition laminar-turbulent regimen. Then the toroidal swirl is not capable to scape further the methane jet as shown in Fig 3, A. It seems that for instance the stream touches the combustion chamber wall and then joins the mean flow. However, the injection velocity increases, as presented in case B $(5 \mathrm{~m} / \mathrm{s})$, and intensifies the methane stream stretch. This may lead to the formation of a Recirculation Zone (RZ) in a high intense swirling flow. The toroidal swirl have a well-defined structure and almost symmetric movement within the mixing species (see figure $3 \mathrm{~B}$ ). The combustion process starts in the vortex interior which is near the oxygen jet. The already mixed species are dragged by the oxygen jet and a well-structured mixing layer is formed with a different velocity.
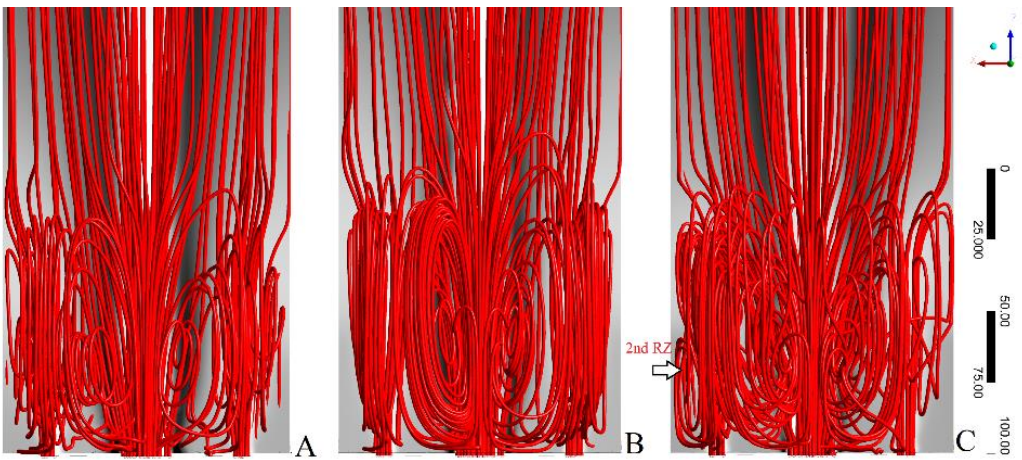

Fig. 3: Stream lines Case A, Case B and Case C from left to right.

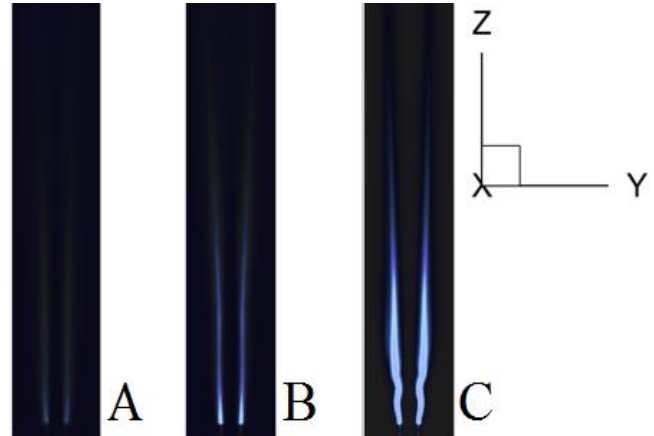

Fig. 4: Perturbations in the Reaction Zone.

The case B stream lines shows a stabilized swirl velocity, which is $34 \%$ greater than the case A and $24 \%$ lower than case $\mathrm{C}$. The mean swirl velocity is from 60 to $70 \%$ of the injection velocity. In the case $\mathrm{C}$, the mixing layer is perturbed by the vortex intensity due to the increased velocity $(10 \mathrm{~m} / \mathrm{s})$. The recirculation zone is chaotic and unstructured swirl flow due to flow jet velocity. The recirculation may lead to an extended perturbation reaching the mean flow. This chaotic structure may affect the reaction zone where the combustion heat is released. Is well known that the mixing layer is easily perturbed by pressure, density or velocity gradient. In all cases (A, B, and C) is by velocity gradient. The velocity difference in the azimuthal direction has a high swirl flow increasing the shear layer strength in $46 \%$, which is higher than case A and 30\% than case B, especially around the boundary of the recirculation. A new RZ can be develop as seen at the lower left corner shown in fig $3 \mathrm{C}$. This new RZ is not as relevant for the mixing process as the larger RZ.

Figure 4 exhibits methane-oxygen mixing layer perturbations at the interface for the next cases: A $\left(0.00367 \mathrm{~L} / \mathrm{D}_{\mathrm{c}}\right), \mathrm{B}$ $\left(0.00435 \mathrm{~L} / \mathrm{D}_{\mathrm{c}}\right)$, and $\mathrm{C}\left(0.00512 \mathrm{~L} / \mathrm{D}_{\mathrm{c}}\right)$ almost methane nozzle diameter. In $\mathrm{A}$ and $\mathrm{B}$ cases, the perturbations are imperceptibles unlike for the case $\mathrm{C}$ where a stretch of the oxygen jet is presented. They are properly observed as wavelike affecting the mixing layer outer structure intensifying the mixing zone between methane and oxygen jets. Figure 5 shows accurately these waves with the tangential velocity contours. This type of waves are directly related with the KH instabilities and can be measured as shown in figure 6 . Therefore, the flame has an instability wave length and frequency as presented in Table 1. 


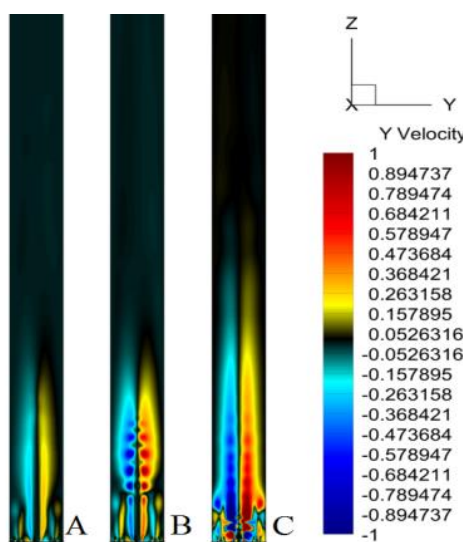

Fig. 5: Tangential Velocity.

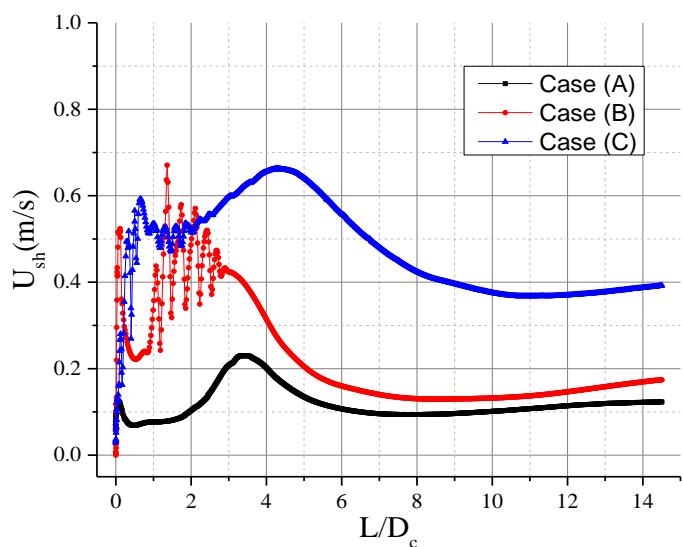

Fig. 6: Velocity fluctuations.

Table 1: Properties of the Kelvin-Helmholtz instabilities.

\begin{tabular}{|c|c|c|c|c|}
\hline Velocity $[\mathrm{m} / \mathrm{s}]$ & Measure distance $[\mathrm{m}]$ & Ridge & $\lambda[\mathrm{m}]$ & $\mathrm{F}[\mathrm{Hz}]$ \\
\hline 2.5 & 0.3048 & 3 & 0.1016 & 24.61 \\
\hline 5 & 0.3048 & 9 & 0.03386667 & 147.68 \\
\hline 10 & 0.3048 & 13 & 0.02344615 & 426.51 \\
\hline
\end{tabular}

\section{Conclusions}

In confined diffusion flames, the recirculation zone is an essential mixture mechanism which has relevant participation on the instabilities development. This type of instabilities do not harm the reaction process but the mixing zone is affected. While the species injection velocity remain between the laminar and transition regimen, the flame instabilities could be produced but the flame front could not be affected. Therefore, the recirculation combined with a strong velocity gradient are the essential mechanisms that leads the KH instabilities.

\section{Acknowledge}

The authors appreciate the financial support provided by National Autonomous University of Mexico, PAPIIT DGAPA IA103515. Also, we appreciate the support provided by the Laboratorio de Ingeniería Térmica e Hidráulica Aplicada, Escuela Superior de Ingeniería Mecánica y Eléctrica del Instituto Politécnico Nacional.

\section{References}

[1] V. R. Katta, W. M. Roquemore, "On the structure of a stretched/compressed laminar flamelet-influence of preferential diffusion," 25th Symp. (Int.) on Combustion - The Combustion Institute, 1994.

[2] F. Takahashi, Gregory T. Linteris, V. R. Katta, "Vortex-coupled oscillations of edge diffusion flames in co-flowing air with dilution," Proceedings of the Combustion Institute, vol. 31, pp. 1575-1582, 2007.

[3] A.J. Yule, N. Chigier, S. Ralph, R. Boulderstone, and J. Ventura, "Combustion-transition interaction in a jet flames," AIAA Journal, vol. 19, pp. 752-760, 1981.

[4] R. Davis, E. F. Moore, W. M. Roquemore, L. D. Chen, V. Vilimpoc, and L.P. Goss, "Preliminary results of a numerical-experimental study of the dynamic structure of buoyant jet diffusion flame," Combustion \& Flame, vol. 83, pp. 263-270, 1991.

[5] R. D. Hancock, F. R. Schauer, and R. P. Lucht, "Thermal Diffusion Effects and Vortex-Flame Interactions in Hydrogen Jet Diffusion Flames," $26^{\text {th }}$ Symposium (International) on Combustion - The Combustion Institute, pp. 1087-1093, 1996.

[6] N. N. Semenov, Some Problems of chemical Kinetics and Reactivity. London: Pergamon Press, 1958.

[7] W.P. Jones, "Models for turbulent flows with variable density and combustion," in Prediction Methods for Turbulent Flows, W. Kollman, Ed. Hemisphere, 1980, pp. 379-421.

[8] Z. Q. Lin, F. Wei, Y. Jin, "Numerical Simulation of Pulverized Coal Combustion and No Formation," Chemical Engineering Science, vol. 58, pp. 5161-5171, 2003. 
[9] P. Warzecha, A. Boguslawski, "LES and RANS modelling of pulverized coal combustion in swirl burner for air and oxy-combustion technologies," Energy, vol. 66, pp. 732-743, 2014.

[10] M. Herrmann, "Numerical Simulation of Turbulent Bunsen Flames With a Level Set Flamelet Model," Combust \& Flame, vol. 145, pp. 357-375, 2006.

[11] M. A. Habib, M. A. Nemitallah, P. Ahmed, M. H. Sharqawy, H. M. Badr, I. Muhammad, M. Yaqub, "Experimental analysis of oxygen-methane combustion inside a gas turbine reactor under various operating conditions," Energy, vol. 86, pp. 105-114, 2015.

[12] M. De la Cruz, G. Polupan, E. Martínez, and I. Carvajal, "Estudio Numérico del Efecto de la Presión en el Proceso de Mezcla Metano-Oxígeno en un Arreglo de Chorros 4-Lug Bolt," Información Tecnológica. CIT Chile, vol. 26, no. 2, pp. 153-162, 2015. 